\title{
APPLICATION OF THE GENERALISED LIKELIHOOD RATIO ALGORITHM TO THE DETECTION OF A BEARING FAULT IN A HELICOPTER TRANSMISSION
}

\author{
F. Antonio Galati ${ }^{\mathrm{a}}$, B. David Forrester ${ }^{\mathrm{a}}$ and Subhrakanti Dey ${ }^{\mathrm{b}}$ \\ ${ }^{a}$ Air Vehicles Division, Defence Science and Technology Organisation, 506 Lorimer Street Fishermans Bend, VIC 3207, \\ Australia. \\ ${ }^{b}$ Department of Electrical and Electronic Engineering, The University of Melbourne, Parkville, VIC 3010, Australia.
}

\begin{abstract}
A Bell 206B main rotor gearbox was run at high load under test conditions in the Helicopter Transmission Test Facility operated by the Defence Science and Technology Organisation (DSTO) of Australia. The test succeeded in initiating and propagating pitting damage in one of the planet gear support bearings. Vibration acceleration signals were recorded periodically for the duration of the test. The time domain vibration signals were converted to angular domain to minimise the effects of speed variations. Auto-Regressive Moving-Average (ARMA) models were fitted to the vibration data and a change detection problem was formulated in terms of the Generalised Likelihood Ratio (GLR) algorithm. Two different forms of the GLR algorithm in window-limited online form were applied. Both methods succeeded in detecting a change in the vibration signals towards the end of the test. A companion paper submitted by the University of New South Wales outlines the corresponding diagnosis and prognosis algorithms applied to the vibration data.
\end{abstract}

Key Words: Generalized Likelihood Ratio, GLR, ARMA, Fault Detection, Bearing Fault, Epicyclic Gear Train, Vibration Analysis

\section{INTRODUCTION}

The vibration characteristics of epicyclic gear trains are more complex than fixed axis gears because the transmission path varies with the rotation of the planet carrier. Since epicyclic gear trains are common in helicopter transmission systems, it is important that suitable data sets be available for testing vibration based fault detection and diagnosis algorithms for helicopter transmission systems. The Helicopter Transmission Test Facility (HTTF) located at the Defence Science and Technology Organisation of Australia is a state of the art facility capable of testing a number of different helicopter transmissions systems at high loads under controlled conditions.

The HTTF was used to create vibration test data corresponding to the generation and propagation of a bearing fault in a Bell 206B-1 helicopter main rotor gearbox. In the first part of this paper, Section 2, the test procedure and outcome of the test is described in detail.

The remainder of the paper is devoted to the application of Generalised Likelihood Ratio (GLR) based fault detection algorithms to the vibration data obtained from the test. Before proceeding it is worthwhile introducing the various notations used throughout the paper. As sequences occur often, a compact subscript notation is used. The notation $y_{(j: k)}$ is used to represent the sequence $y_{j}, y_{j+1}, \ldots, y_{k}$ when $k>j$, and $y_{j}, y_{j-1}, \ldots, y_{k}$ when $k<j$. The convention of using uppercase letters for random variables and lowercase letters for the values of random variables is also adopted here. The symbol $\mathbb{R}$ is used to denote the set of Real numbers.

The general model used in statistical change detection is a (chronological) sequence of random variables or vectors, $\left\{Y_{k}\right\}$, $k=0,1,2, \ldots$, with known parametrised conditional density $p_{\theta}\left(y_{k} \mid y_{(k-1: 1)}\right)$. The parameter, $\theta$, is constant (or nearly so) and equal to $\theta_{0}$ before some unknown change time $t_{0}$ (i.e. for all $k<t_{0}$ ). At the change time, $\theta$ changes abruptly to a new value $\theta_{1}$ where it remains for all time $k>t_{0}$. A typical change detection algorithm comprises a suitable test statistic, $S_{k}$, a decision function, $g_{k}$, which usually converts the test statistic into a form in which a suitable alarm threshold can be established, and an alarm time, $t_{a}$, to indicate when the decision function exceeds the chosen threshold. 
As the problem formulation in statistical change detection is very general, the scope for applications is wide. Example applications include gain updating in adaptive tracking algorithms, signal segmentation for tasks such as speech recognition, and quality control (which includes fault detection). A comprehensive introduction to statistical change detection theory and applications can be found in the book [1].

The GLR is a powerful test statistic for use in change detection algorithms, however a well known problem with the online application of the GLR algorithm is that the number of operations to perform at each time step, $k$, grows with $k$ to infinity. This difficulty comes about because the Likelihood ratio cannot be computed recursively. By restricting the algorithm to a data window of fixed size, the computational burden can be reduced to a manageable level, but the resulting window-limited algorithm becomes sub-optimal. This idea was first investigated in [2], in the context of detecting additive changes in linear state space systems.

It was shown in [3] that the performance of the windowlimited GLR algorithm depends critically on the choice of window parameters, even in the simple case of detecting additive changes in white noise. The choice of window parameters also affects the choice of decision threshold which further complicates the issue. A number of authors $[4,5,6]$ have addressed the issue of finding optimal window parameters for the case of additive changes in linear systems, however we are concerned here with the more difficult problem of detecting spectral (non-additive) changes in a signal, and in particular to spectral changes in an ARMA model.

In [3], a novel decision function for the window-limited GLR algorithm is introduced which is shown through simulations to be more robust against sub-optimal choices of window parameters. In the second part of this paper we compare the performance of the window-limited GLR algorithm with the new decision function to that of the window-limited GLR algorithm using the conventional decision function. In Section 3 the change detection problem is formulated in terms of the GLR algorithm, and in Section 4 the results are presented and discussed in detail.

\section{TEST DESCRIPTION AND OUTCOME}

The Bell 206B-1 main rotor gearbox, shown in Figure 1, comprises an input bevel and pinion, driven by the engine, and a single stage epicyclic reduction unit. The main rotor is driven through the planet carrier. There exist both three and four planet variants for the Bell 206 gearbox. The three planet variant was used for this test. Accelerometers were mounted on the gearbox casing in the vicinity of the pinion and bevel gear mesh and in the vicinity of the epicyclic ring gear. The input shaft also contained a tachometer producing one pulse per revolution of the input shaft. The tachometer signal was used to convert the vibration signals from time domain to angular domain.

The aim of the test was to initiate and propagate a non-seeded fault in the gearbox. In order to achieve the aim in a reasonable amount of time, the gearbox was run at 150 percent of it's rated operating load, with periodic reductions in load to facilitate the recording of vibration signals. Figure 2 shows the load regime that was repeated every thirty minutes throughout the test.

The planet support bearing contains two rows of convex rollers and an outer race which is integral with the planet gear. The bearing sustained fatigue spalling and subsequent pitting damage to the inner race, outer race, and rolling elements. The majority of damage occurred to the inner race. This can be explained in part by the fact that the inner race does not rotate as the carrier rotates, therefore the load is concentrated in the same part of the inner race rather than being distributed around the whole inner race over time. Figure 3 shows the inner race of the planet bearing in which the pitting damage can be seen clearly in both the upper and lower tracks, with the majority of damage to the lower track.

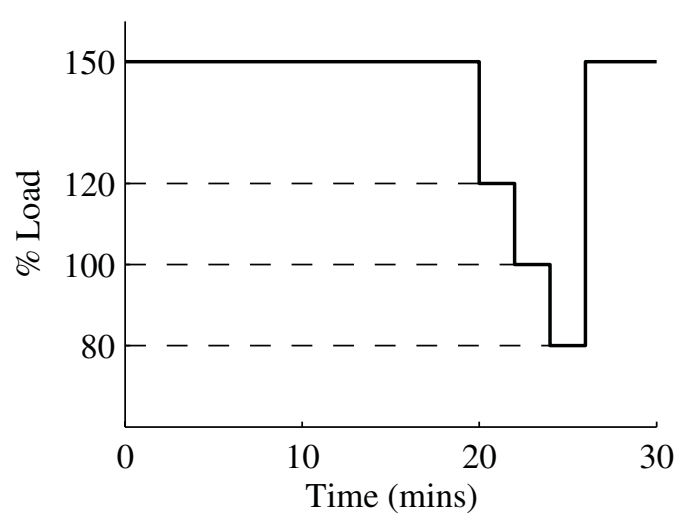

Figure 2: Gearbox load regime repeated throughout the test. 